\title{
Kataraktoperationen bei Uveitis
}

\author{
Jakob Schweighofer · Yosuf El-Shabrawi
}

Eingegangen: 15. Juli 2020 / Angenommen: 4. August 2020 / Online publiziert: 3. September 2020

(C) Der/die Autor(en) 2020

Zusammenfassung Die Katarakt stellt bis heute eine maßgebliche Ursache für Visusverlust bei Patienten mit einer Entzündung der Uvea dar. Mehrere Studien der letzten Jahre zeigten hier zwar, dass eine Kataraktoperation eine Verbesserung des Visus erzielen kann, die Prognose jedoch im Vergleich zu einer normalen Patientengruppe stark reduziert ist.

Schlüsselwörter Uveitis - Katarakt . Kataraktoperation · Entzündung · Therapie

\section{Cataract surgery in uveitis}

Summary Up to now cataract represents a leading cause of loss of visual acuity in patients with inflammation of the uvea. Several studies in recent years have shown that cataract surgery can achieve an improvement in visual acuity; however, the prognosis is greatly reduced in comparison to a normal patient group.

Keywords Uveitis · Cataract · Cataract surgery · Inflammation · Therapy

\section{Katarakt und Uveitis}

Bei der Uveitis handelt es sich um die dritthäufigste Erblindungsursache weltweit. Insbesondere die Katarakt spielt hier eine bedeutende Rolle, da es bei bis zu $40 \%$ dieser Patientengruppe zu einem Visusverlust

\section{Dr. J. Schweighofer}

Augenabteilung, Stadtspital Triemli Zürich,

Birmensdorferstr. 497, 8063 Zürich, Schweiz

Prim. Univ. Prof. Dr. Y. El-Shabrawi $(\varangle)$

Klinikum Klagenfurt, Feschnigstr. 11, 9020 Klagenfurt, Österreich

Medical University of Graz, Graz, Österreich yosuf.elshabrawi@kabeg.at ausschließlich oder besonders durch die Entstehung einer Linsentrübung kommt [1].

Aufgrund des chronischen Entzündungsprozesses und der häufig notwendigen lokalen Steroidapplikation kommt es hier bereits früh zur Entwicklung einer Katarakt. Zwar führt eine chirurgische Intervention in den meisten Fällen zu einer anschließenden Verbesserung des Visus, jedoch ist die Prognose bis heute aufgrund mehrerer Faktoren stark reduziert. Sowohl mögliche intraoperative als auch postoperative Komplikationen führen dazu, dass die postoperativen Visusergebnisse nach einer Phakoemulsifikation bis heute nicht überzeugend sind.

In einem Review aus dem Jahr 2014 wurde gezeigt, dass der Anteil jener Uveitispatienten, die nach einer Kataraktoperation einen Visus von $\geq 0,5$ hatten, lediglich bei knapp $68 \%$ lag [2]. Noch gravierender wird dieses Ergebnis, wenn man berücksichtigt, dass es sich hier um Patienten handelt, deren Uveitis sich seit mindestens 2 Monaten in vollständiger Remission befand. Bei Patienten mit aktiver Uveitis zum Zeitpunkt der Operation zeigten sich noch schlechtere Ergebnisse. Hier kam es nur in $23 \%$ der Fälle zu einem postoperativen Visusanstieg von $\geq 0,5$.

Diese Daten konnten in einer großen Studie aus dem Jahr 2017 bestätigt werden. Hier wurden die Ergebnisse der Kataraktoperationen von 1173 Uveitispatienten mit jenen von 95.573 normalen Kataraktfällen verglichen [3]. Hier zeigte sich ebenfalls, dass die Visusergebnisse bei Uveitispatienten signifikant schlechter waren als jene der altersbedingten Kataraktpatienten $(0,4 \pm 0,25$ vs. $0,6 \pm 0,3)$. Des Weiteren zeigte sich in dieser Studie, dass es bei 3,8\% der Uveitispatienten sogar $\mathrm{zu}$ einem signifikanten Visusverlust im Vergleich zu den präoperativen Visusdaten kam. Hier muss jedoch berücksichtigt werden, dass dies statistisch gesehen keinen Unterschied zur Kontrollgruppe darstellt (2,9\%). 
Ursachen bzw. Erklärungen für die signifikant schlechtere Visusprognose sind mannigfaltig. Intraoperative Komplikationen belaufen sich häufig auf hervorgerufene Probleme der chronischen Entzündung. Insbesondere Synechien und hierbei notwendige zusätzliche operative Schritte sind hier zu erwähnen.

Für den postoperativen Verlauf ist besonders die erhöhte Gefahr eines Makulaödems hervorzuheben. Hier liegt die Inzidenz bei bis zu $21 \%$ [4]. Bei Patienten mit einer aktiven Uveitis liegt die Inzidenz eines Ödems im ersten postoperativen Monat sogar bei 38, nach 3 Monaten bei $8 \%$ [5]. Für diesen signifikanten Unterschied zur Kontrollgruppe ist vermutlich das erhöhte Level an Entzündungsmediatoren nach der durchgeführten Operation ursächlich. Selbst bei gesunden Patienten kommt es bereits zu einem starken Anstieg an Interleukinen, besonders IL-8, in den ersten $18 \mathrm{~h}$ nach dem chirurgischen Eingriff [6]. Da bei Patienten mit einer Uveitis das Level an Chemokinen bereits präoperativ um mehr als das 7 -Fache erhöht ist, führt der weitere Anstieg an Entzündungsmediatoren schnell zu postoperativ entzündlich assoziierten Komplikationen [7].

Das primäre Ziel im präoperativen Zeitraum besteht grundsätzlich im Verhindern bzw. Hinauszögern einer Katarakt in der betroffenen Patientengruppe. Dies gelang der Gruppe von Foster et al. In einer damals durchgeführten Studie war es ihnen möglich die Inzidenz einer Katarakt unter Einsatz von nichtsteroidalen Immunmodulatoren bei Kindern mit juveniler idiopathischer Arthritis in einem Follow-up von 24 Jahren auf $18 \%$ zu reduzieren [8].

Im Vergleich dazu liegt die Inzidenz der Katarakt bei Juveniler Idiopathischer Arthritis im Großteil der Studien bei rund $50 \%$ der Kinder oder Jugendlichen. [9].

Somit zeigt sich, wie wichtig der frühzeitige Einsatz von Immunmodulatoren wie Methotrexat, Mycophenolat-Mofetil oder die Verwendung von Biologika ist, um die Langzeitprognose von Uveitispatienten signifikant zu verbessern [10].

Sollte es nun bei Uveitispatienten zu einer operationswürdigen Katarakt gekommen sein, sollte präoperativ erneut eine komplette Abklärung der Genese erfolgen. Insbesondere der Ausschluss infektiöser Ursachen der intraokulären Entzündung spielt hierbei eine wichtige Rolle. Dies, weil z. B. gezeigt werden konnte, dass es bei Patienten mit okulärer Toxoplasmose innerhalb der ersten 4 Monate in $30 \%$ zu einer Reaktivierung der Uveitis nach Kataraktoperation kam [11].

Im Rahmen von herpetischer Uveitis zeigten sich im postoperativen Follow-up von 2 Jahren schwere Komplikationen wie die Reaktivierung der Keratouveitiden, persistierende Epitheldefekte und chronisch zystoide Makulaödeme in 54\% aller Patienten [12].

Die Wahl der richtigen Therapie ist hier für das Ergebnis der Operation unerlässlich. Bei Patienten mit einer bekannten rezidivierenden Toxoplasma-assoziierten Uveitis sollte präoperativ eine Therapie mit Clindamycin (300 mg 4-mal täglich) über den Zeitraum von bis $\mathrm{zu} 1$ Woche verabreicht werden. Bei Patienten mit einer bekannten herpetischen Uveitis empfiehlt sich eine Therapie mit antiviralen Therapeutika wie Aciclovir (400 mg 3-mal täglich) oder Valaciclovir (500 mg 2-mal täglich) präoperativ für 1 Woche [13].

Die präoperativ angesetzte antivirale Therapie sollte unbedingt im postoperativen Verlauf fortgeführt werden. Insbesondere bei einer Herpes-Zoster-assoziierten rezidivierenden Uveitis ist eine Therapie unter Umständen sogar für bis zu 2 Jahre zu belassen, um eine mögliche Reaktivierung der Entzündung zu verhindern [12].

Im Fall einer vorliegenden nichtinfektiösen Uveitis ist v. a. darauf zu achten, dass eine vollständige Remission der intraokulären Entzündung für zumindest 3 Monate präoperativ erreicht wird. Hier empfiehlt es sich, außerdem eine präoperative Therapie 1 Woche vor Eingriff mit Prednisolonacetat-Tropfen 6- bis 8-mal täglich einzuleiten. Ergänzt sollte diese Therapie noch um lokale nichtsteroidale Antiphlogistika werden (2- bis 4-mal täglich für 1 Woche).

Auch wird eine systemmische Therapie mit Steroiden $(0,5-2 \mathrm{mg} / \mathrm{kg})$ präoperativ für einen Zeitrahmen von ca. 1 Woche empfohlen. In einer Studie wurde die Effektivität einer 2-wöchigen präoperativen oralen Prednison-Gabe mit 0,5 mg/kg pro Tag mit einer 1-maligen präoperativen intravenösen Methylprednisolon-Gabe mit $15 \mathrm{mg} / \mathrm{kg}$ verglichen. Es zeigte sich, dass die 2-wöchige orale, niedrig dosierte Steroidgabe der intravenösen Gabe signifikant überlegen war. Damit wird auch weiterhin die orale Steroidtherapie präoperativ bei Uveitispatienten empfohlen [13]. Insbesondere auf die Inzidenz des Makulaödems wirkt sich die Installation einer solchen oralen Steroidtherapie positiv aus [5].

Ebenfalls ist eine ausreichende Behandlung mit Immunmodulatoren präoperativ zu überlegen. Da allerdings je nach Substanz der mittlere Zeitraum bis zum Therapieerfolg zwischen 4 und 6 Monaten liegt, ist eine entsprechende Planung der Behandlung notwendig [14].

Alternativ zu oralen oder intravenösen Steroiden kann eine präoperative Therapie mit Steroidimplantaten überlegt werden. So zeigte sich in der MUST-Studie bei Patienten mit einem Fluocinolon-Implantat, im Vergleich zu jenen, die präoperativ eine Stand-ofCare erhalten haben, ein signifikant höherer Visusanstieg 1 Monat bzw. auch 3 Monate postoperativ. Auch war die Wahrscheinlichkeit einer Reaktivierung der Uveitispatienten in der Fluorchinolone-Gruppe signifikant geringer als in der Stand-of-care-Patientengruppe $(26,5 \%$ vs. $44,4 \%, p=0,043)$ [15].

Häufiger angewendet werden natürlich Dexamethason-Implantate. In einer Studie wurden $17 \mathrm{Au}$ gen/14 Patienten mit einem präoperativ erkennbaren 
uveitischen-CME inkludiert. Es zeigte sich in keinem der Augen präoperativ eine entzündliche Aktivität. Wurde das Implantat $\leq 4$ Wochen (8 Augen) eingesetzt, so zeigte sich postoperativ eine mittlere Abnahme der CMT um 47,0 $\mu \mathrm{m}$. Wurde die Kataraktoperation allerdings $\geq 4$ bis 12 Wochen (9 Augen) nach der Dexamethason-Gabe durchgeführt, so zeigte sich postoperativ eine durchschnittliche Zunahme der CMT um 51,1 $\mu \mathrm{m}$ $(p=0,005)$.

Damit wird empfohlen, die Dexamethason-Gabe in einem Zeitraum von $\leq 2$ Wochen vor der geplanten Kataraktoperation durchzuführen [16].

Für die intraoperative Therapie bzw. Prophylaxe eines möglichen Entzündungsrezidivs besteht ebenfalls die Möglichkeit der Implantation eines Dexamethason-Implantats, sollte diese präoperativ noch nicht erfolgt sein. Ebenfalls kann die Verabreichung von Triamcinolon in Betracht gezogen werden. Hier empfiehlt sich eine intravitreale Gabe welche sich in einer Studie einer peribulbären Verabreichung als überlegen herausstellte [17].

Die implantierte Linse selbst spielt für den postoperativen Verlauf und für das Risiko eines Rezidivs keine Rolle. Dies konnte durch eine im Jahre 2002 publizierte Studie gezeigt werden. In jener konnte kein signifikanter Unterschied zwischen einzelnen Linsen (Hydrophil, Hydrophob oder Silikon) nachgewiesen werden [18].

Postoperativ ist es wichtig, die antiinflammatorische Therapie für zumindest 6 bis 8 Wochen beizubehalten. Bei bekanntem Glaukom sollte 1-2 h vor der Operation eine orale Gabe von Acetazolamid überlegt werden, um unmittelbar postoperative Augendruckanstiege $\mathrm{zu}$ verhindern. Auch sollte mit der intensiven lokalen Steroidtherapie unmittelbar postoperativ begonnen werden und nicht erst am ersten Tag danach. Topische NSAIDs werden wie präoperativ 2- bis 4-mal täglich empfohlen, und die oralen Steroide $(1 \mathrm{mg} / \mathrm{kg} / \mathrm{Tag})$ für 1 Woche, dann je nach Verlauf kann um $10 \mathrm{mg} /$ Woche über 4 bis 6 Wochen reduziert werden.

Abschließend ist es wichtig hervorzuheben, dass trotz der erhöhten Inzidenz intraoperativer oder postoperativer Komplikationen und einer reduzierten Visusprognose eine operative Sanierung der Katarakt bei Uveitispatienten in einer großen Anzahl zu einer Verbesserung des Visus und damit der Lebensqualität des Patienten führt und daher indiziert ist [3].

Funding Open access funding provided by Medical University of Graz.

Interessenkonflikt J. Schweighofer und Y. El-Shabrawi geben an, dass kein Interessenkonflikt besteht.

Open Access Dieser Artikel wird unter der Creative Commons Namensnennung 4.0 International Lizenz veröffentlicht, welche die Nutzung, Vervielfältigung, Bearbeitung, Verbreitung und Wiedergabe in jeglichem Medium und Format erlaubt, sofern Sie den/die ursprünglichen Autor(en) und die Quelle ordnungsgemäß nennen, einen Link zur Creative Commons
Lizenz beifügen und angeben, ob Änderungen vorgenommen wurden.

Die in diesem Artikel enthaltenen Bilder und sonstiges Drittmaterial unterliegen ebenfalls der genannten Creative Commons Lizenz, sofern sich aus der Abbildungslegende nichts anderes ergibt. Sofern das betreffende Material nicht unter der genannten Creative Commons Lizenz steht und die betreffende Handlung nicht nach gesetzlichen Vorschriften erlaubt ist, ist für die oben aufgeführten Weiterverwendungen des Materials die Einwilligung des jeweiligen Rechteinhabers einzuholen.

Weitere Details zur Lizenz entnehmen Sie bitte der Lizenzinformation auf http://creativecommons.org/licenses/by/4. $0 /$ deed.de.

\section{Literatur}

1. Durrani OM, Tehrani NN, Marr JE, Moradi P, Stavrou P, Murray PI. Degree, duration, and causes of visual loss in uveitis. Br JOphthalmol. 2004;88(9):1159-62.

2. Mehta S, Linton MM, Kempen JH. Outcomes of cataract surgery in patients with uveitis: a systematic review and meta-analysis. Am JOphthalmol. 2014;158(4):676-692.e7.

3. Chu CJ, Dick AD, Johnston RL, Yang YC, Denniston AK. Cataract surgery in uveitis: a multicentre database study. $\mathrm{Br}$ JOphthalmol.2017;101(8):1132-7.

4. Ram J, Gupta A, Kumar S, Kaushik S, Gupta N, Severia S. Phacoemulsification with intraocular lens implantation in patients with uveitis. J Cataract Refract Surg. 2010;36(8):1283-8.

5. Bélair ML, Kim SJ, Thorne JE, Dunn JP, Kedhar SR, Brown DM, et al. Incidence of cystoid macular edema after cataract surgery in patients with and without uveitis using optical coherence tomography. Am J Ophthalmol. 2009;148(1):128-135.e2.

6. Tu KL, Kaye SB, Sidaras G, Taylor W, Shenkin A. Effect of intraocular surgery and ketamine on aqueous and serum cytokines. Mol Vis. 2007;13:1130-7.

7. Valentincic NV, de Groot-Mijnes JD, Kraut A, Korosec P, Hawlina M, Rothova A. Intraocular and serum cytokine profiles in patients with intermediate uveitis. Mol Vis. 2011;17:2003-10.

8. Ceisler EJ, Foster CS. Juvenilerheumatoid arthritis anduveitis: minimizing the blinding complications. IntOphthalmol Clin. 1996;36(1):91-107.

9. Skarin A, Elborgh R, Edlund E, Bengtsson-Stigmar E. Longterm follow-up of patients with uveitis associated with juvenile idiopathic arthritis: a cohort study. Ocul Immunol Inflamm. 2009;17(2):104-8.

10. Galor A, Jabs DA, Leder HA, Kedhar SR, Dunn JP, Peters GB 3rd, et al. Comparison of antimetabolite drugs as corticosteroid-sparing therapy for noninfectious ocular inflammation. Ophthalmology. 2008;115(10):1826-32.

11. Bosch-Driessen LE, Berendschot TT, Ongkosuwito JV, Rothova A. Ocular toxoplasmosis: clinical features and prognosis of 154 patients. Ophthalmology. 2002;109(5):869-78.

12. He Y, de Melo Franco R, Kron-Gray MM, Musch DC, Soong HK. Outcomes of cataract surgery in eyes with previous herpes zoster ophthalmicus. J Cataract Refract Surg. 2015;41(4):771-7.

13. Van Gelder RN, Leveque TK. Cataract surgery in the setting of uveitis. Curr Opin Ophthalmol.2009;20(1):42-5.

14. Meacock WR, Spalton DJ, Bender L, Antcliff R, Heatley C, Stanford MR, et al. Steroid prophylaxis in eyes with 
uveitis undergoing phacoemulsification. Br J Ophthalmol. 2004;88(9):1122-4.

15. Sheppard JD Jr., Nguyen QD, Usner DW, Comstock TL. Postcataract outcomes in patients with noninfectious posterior uveitis treated with the fluocinolone acetonide intravitreal implant. Clin Ophthalmol. 2012;6:79-85.

16. Larochelle MB, Smith J, Dacey MS. Dexamethasone intravitreal implant in the treatment of uveitic macular edema in the perioperative cataract setting: a case series. Am J Ophthalmol.2016;166:149-53.

17. Roesel M, Tappeiner C, Heinz C, Koch JM, Heiligenhaus A. Comparison between intravitreal and orbital floor triamcinolone acetonide after phacoemulsification in patients with endogenous uveitis. Am J Ophthalmol. 2009;147(3):406-12.

18. Abela-Formanek C, Amon M, Schild G, Schauersberger J, Kolodjaschna J, Barisani-Asenbaum T, et al. Inflammation after implantation of hydrophilic acrylic, hydrophobic acrylic, or silicone intraocular lenses in eyes with cataract and uveitis: comparison to a control group. J Cataract RefractSurg. 2002;28(7):1153-9.

Hinweis des Verlags Der Verlag bleibt in Hinblick auf geografische Zuordnungen und Gebietsbezeichnungen in veröffentlichten Karten und Institutsadressen neutral. 